\title{
New Vision Sensor to Measure and Monitor Gas Pressure
}

\author{
K. MuRAWSKI* \\ Military University of Technology, Institute of Teleinformatics and Automatics, \\ S. Kaliskiego 2, 00-908 Warsaw, Poland \\ (Received May 12, 2014; in final form April 29, 2015)
}

\begin{abstract}
The paper presents the construction and the use of a video sensor developed to measure air pressure. A characteristic feature of the device is pressure measurement based on digital image processing and self-diagnostics performed on the basis of an image. The paper presents the results of sensor calibration and measurements taken during the tests. It has been shown that the developed video-manometer model that is equipped with a flat diaphragm allows measuring the air pressure in the range of $0-100$ mbar with an error less than $2 \%$.
\end{abstract}

DOI: 10.12693 /APhysPolA.128.6

PACS: 06.30.Bp, 07.05.Pj, 07.07.Df

\section{Introduction}

Pressure sensors play an important role in the control of technological processes. They ensure safe operation of devices and also enable operation of autonomous control systems and the surveillance of technical systems. They are found in almost every branch of the industry. They can be found in the food, chemical, fuel, civil and military aviation industry, in medicine $[1-5]$ and in others. They are also used in research and development works [6] as well as in diagnostics. Due to the large area of applications and the range of measured pressures, a significant number of pressure sensors and transducer structures have been developed. The direct measurement of the pressure is performed using: diaphragm, Bourdon tube, strain gauge diaphragm, capacitance diaphragm, $\mathrm{Hg}$ manometer, liquid manometer, radioactive gauge, McLeod compression manometer, U-tube manometer. The pressure is measured indirectly, when the properties of gases are known, using: momentum transfer viscosity (quartz fiber, rotating), energy transfer - thermal conductivity (thermocouple, Pirani, thermistor, convection gauge), charge generation with hot cathode (triode ion, Bayard-Alpert ion, Schulz-Phelps ion, extractor ion) and cold cathode (magnetron, inverted magnetron, double inverted magnetron) as well as mass spectrometer [7]. These techniques cover the pressure measuring range from $1.33 \times 10^{-15}$ bar to 10 bar [7].

Today, a large emphasis is placed on the construction of intelligent pressure sensors. Such sensors, in addition to the sensing element, include electronics systems required for the linearisation of the sensor characteristics, to eliminate the effects of temperature, autocalibration and measurement range and reference points (zero and alarm thresholds) settings. The observed sensors to measure the pressure of gases with the optical path measurement fit the trend. The following solutions have been

*e-mail: k.murawski@ita.wat.edu.pl

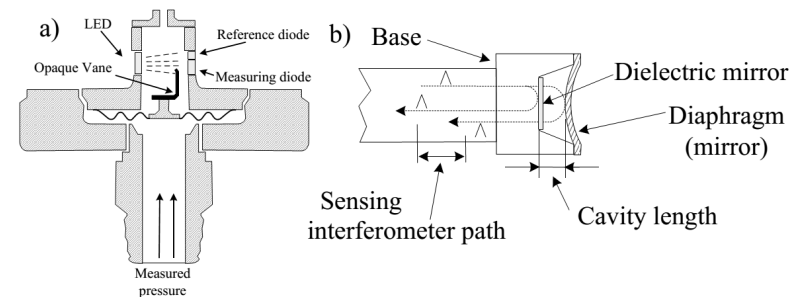

Fig. 1. Sensors for measuring pressure with the optical path [5].

noted in the literature. The first uses two photodiodes and one IR LED diode [8]. In the considered arrangement, Fig. 1a, the LED diode along with one of the photodiodes forms a slotted optocoupler. The second photodiode produces a reference signal used to stabilize the sensor operation. An addition to the optical path is the corrugated diaphragm and the shutter (blade). With the increase in the measured pressure, the diaphragm slightly deforms below $0.5 \mathrm{~mm}$ [7], while lifting the blade. The blade blocks the path of light, whose intensity is measured. Sensors of this type are available for ranges from 0.35 bar to 4.13 bar, assuring a measurement accuracy of about $0.1 \%$. The second solution, Fig. 1b, consists of using an optical fiber with a diaphragm measuring head at the end [9]. The gas pressure is measured using the correlation between the gas pressure applied and the intensity of light reflecting from the surface of the diaphragm [9]. The width of the optical cavity located between the diaphragm and the optical fiber is also often measured [10]. This distance is determined using a Fabry-Perot interferometer, the operation is discussed in [11]. The pressure measurement result is presented as a value of DC voltage in a range from $0 \mathrm{~V}$ to $5 \mathrm{~V}$ or $0 \mathrm{~V}$ to $10 \mathrm{~V}$ [10]. The measurement result can also be expressed in the form of a current intensity from $4 \mathrm{~mA}$ to $20 \mathrm{~mA}[12,13]$. Pressure measurement using fiber optic Bragg grating sensors is also a known solution. The solution presented in [14] allowed realising the measurement of pressure in the range from 0 bar to 1.8 bar with a measurement error percentage of $3.13 \%$. 


\section{Motivation}

The aim of this work was to develop a videomanometer and a practical application of a new method of measuring pressure. The proposed method is based on visual measuring in changes in the width of the marker placed on the surface of the deformable diaphragm. The essence of this method is to determine the distance to the diaphragm on the basis of size of blur of the marker view placed on her surface. The proposed technique belongs to a group of methods that in the field of computer science as well as computer vision is called as technique of calculation "depth from focusing and defocusing". These methods are violently developed since long time. Currently to the measurement of distance the contrast images as well as blurry images are frequently used. Among the methods belonging to the "depth from defocus" stand out those that require at least of two images taken with different but known focus settings [15], method supported by the model of image blurring [16] and techniques similar to used in this paper, in which the distance to an object or a depth map is determined on the basis of a single image [17]. A characteristic feature of the used technique is that the calculated distance is determined directly from the blur size of the marker view. Thereby the distance is determined in real time. It is a completely different approach than presented in [17], where the calculated distance is determined on basis of the image view and inhomogeneous reverse heat equation.

The main advantages of the presented technique is also non-contact measurement; galvanic isolation of the sensor from the chamber of increased and/or decreased pressure; high sensitivity, reproducibility and accuracy in carried out measurements; interference resistant; lack of frictional and mechanical components; design simplicity; small and scalable sensor sizes; the ability to measure the pressure of gases using diaphragms: flat, corrugated, bellows and capsulated; low-cost implementation; the possibility of using video fiber optics; real-time visual sensor operation self-diagnostics.

Pressure sensors that use the principles of optics exhibit optimum properties for their use in explosive and medical environments. The proposed sensor design extends the already known properties of optical sensors with the real time vision self-diagnostics function. The developed video-manometer can be used in medicine, for example, or in control systems and gas transportation systems.

\section{Construction of the video-manometer}

The active element in the made video-manometer was a white silicone flat diaphragm with a thickness of $0.5 \mathrm{~mm}$, Fig. 2. The diaphragm was placed in a modified 2 N25 solenoid valve, Fig. 3 and Fig. 4. A black circular marker with a diameter of $6 \mathrm{~mm}$ was pasted in the central part of the diaphragm. The marker was monitored by a board camera with a mounted visible light filter and a lens with a fixed focal length of $f=12 \mathrm{~mm}$. The distance from the front of the lens to the surface of the diaphragm was $60 \mathrm{~mm}$. The camera in the constructed model, worked in IR mode with a resolution of $640 \times 480$ pixels and at $60 \mathrm{fps}$. The marker was illuminated with IR LED diodes with a wavelength of $\lambda=850 \mathrm{~nm}$, where the $I_{\mathrm{F}}$ current was stabilized using the technique given in $[18,19]$.

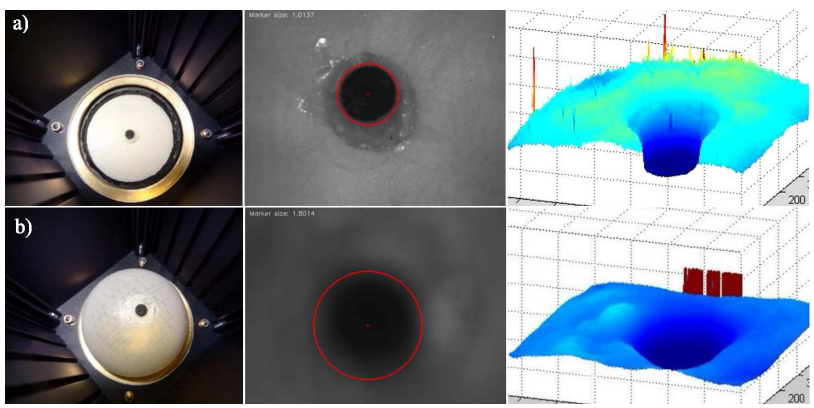

Fig. 2. View of the diaphragm (left), marker (middle) and the pixel brightness distribution (right) for flat diaphragm (a) and corrugated diaphragm (b).

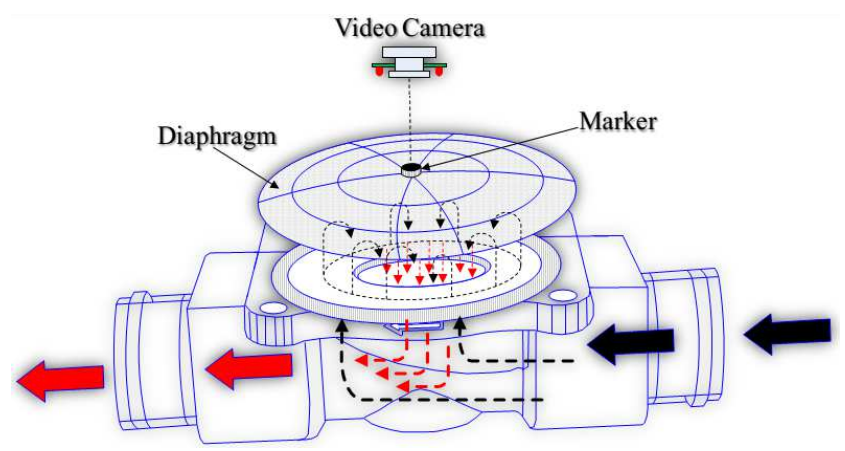

Fig. 3. The scheme and view of the video manometer.

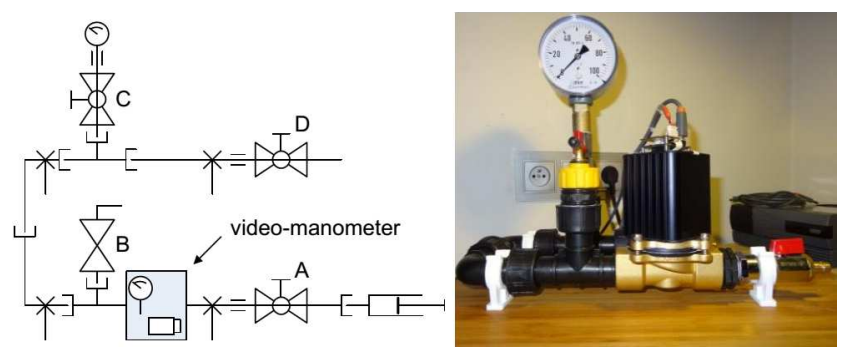

Fig. 4. The diagram and the view of the measuring station for calibrating and testing the video-manometer.

The correlation between the measured width of the marker and the measured air pressure was used for pressure measurement. The applied pressure changes the distance of the marker to the front of the camera lens. As a result, the focus in the images becomes blurred. The image underwent segmentation by using digital image processing techniques [20-22]. As a result of image processing the view of the marker was isolated, which width constitutes the basis for determining the value of the applied pressure. The $W$ marker width varies according to the principle: pressure increases $\rightarrow$ blurry image, 
$W$ increases, Fig. 2b; pressure without changes $\rightarrow$ sharp image, $W$ does not change, Fig. 2a; pressure decreases (underpressure) $\rightarrow$ image blurred, $W$ decreases.

The measurement of the width of the marker was performed using the technique presented in [23]. The operation of the developed sensor was examined by measuring the air pressure in the range of 0 mbar to 100 mbar. The model used a flat diaphragm. The choice of the shape of the diaphragm was dictated by the unavailability of the corrugated, bellows or capsules diaphragm. As a result, considerable deformations of the diaphragm shape were observed, Fig. 2b. Ultimately, the average air pressure measurement error was $1.718 \%$. The results of the measurement technique used for measuring the width of the marker indicate that the use of a corrugated diaphragm or bellows should allow for pressure measurement with an error below $1 \%[24,25]$.

\section{Measurement system configuration, video-manometer calibration procedure}

The developed video-manometer was tested at the measuring station shown in Fig. 4. The measuring station is equipped with a pressure manometer with a range of 100 mbar and class 1.6 as well as manually closed valves $A-D$, where $A$ intake valve, $D$ outlet valve. Valve $C$ is mounted in front of the calibration manometer. It is used to disconnect the manometer, when the video-manometer is tested in conditions of underpressure. Valve $B$ acts as an auxiliary. Valves $A$ and $D$ were most commonly used in the experiments. Valve $A$ was used to supply air and to determine the pressure value. Calibration and pressure measurement was performed with valve $D$ closed. The calibration procedure consisted in forcing pressure values in the range from 0 mbar to 100 mbar in steps of 2 mbar. For each pressure setting 1000 measurements of the marker width were made, from which the average minimum and maximum was calculated. The relation

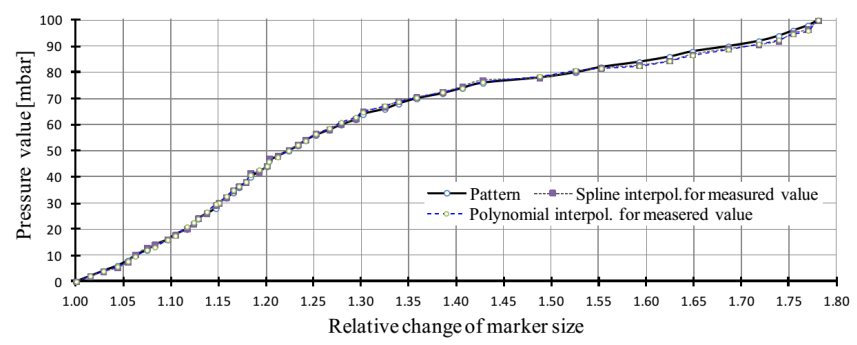

Fig. 5. The measured pressure as a function of relative changes in average marker sizes.

between exerted air pressure values and average changes in the width of the marker is shown in the graph Pattern, Fig. 5. Lagrange interpolation and cubic spline interpolation parameters were determined for the average values of the width of the marker. The values of exerted pressure were recreated on their basis for the obtained average values of the width of the marker. The calculation results are shown on graphs Spline and Polynomial, Fig. 5.

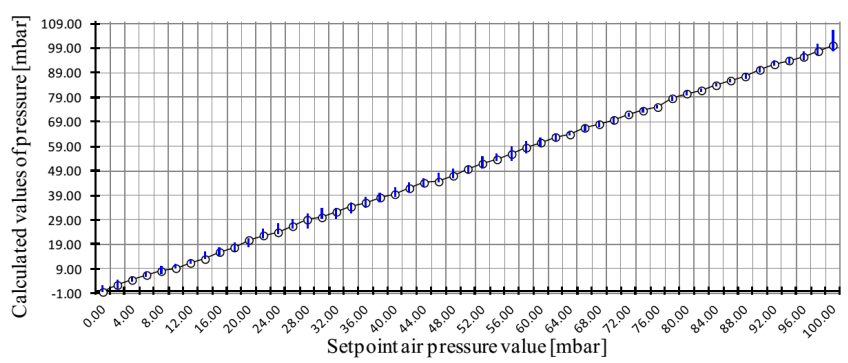

Fig. 6. The dispersion of the measured pressure obtained during calibration.

The estimated dispersion of pressure measuring results made using the described method is illustrated in Fig. 6. The dispersion of the determined pressure values are due to random errors that occur during the measurement of the width of the marker. The average measurement error equaled 0.396 mbar. The maximum pressure measurement error during calibration was equal to 1.6 mbar.

\section{Research results}

Video-manometer was tested in the arrangement as presented in Fig. 4. The air pressure values were applied in steps of 2 mbar in the range of 0 mbar to 100 mbar. A total of ten series of measurements were made, 50 pressure settings each. Measurement relative error $\delta$ and absolute error $|\Delta P|$ were determined for each series. Measurement errors were assessed separately for the Lagrange interpolation $\left(\delta_{\mathrm{W}},\left|\Delta P_{\mathrm{W}}\right|\right)$ as well as the cubic spline interpolation $\left(\delta_{\mathrm{S}},\left|\Delta P_{\mathrm{S}}\right|\right)$. The obtained average error values for the applied pressure settings are presented in Fig. 7. Higher error values were obtained in

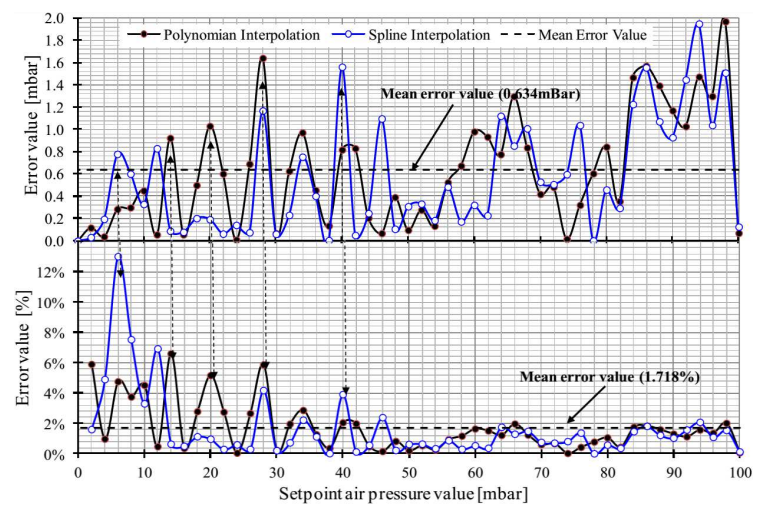

Fig. 7. Average absolute and proportional measurement error.

the experiment for the Lagrange interpolation. The average absolute measurement error in the study for the entire measurement range equaled 0.634 mbar. In comparison, this error for the cubic spline interpolation was at a level of 0.562 mbar. The maximum pressure measurement error values in the entire range of measurement stood at 1.972 mbar for the Lagrange interpolation and 1.952 mbar for the cubic spline interpolation. These errors result from a worse adjustment of the model based 
on the Lagrange interpolation for the pressure values of 98 mbar and 94 mbar for the cubic spline interpolation. After limiting the range to 90 mbar the absolute maximum measurement error equaled 1.6 mbar. In terms of percentage, the average measurement error within the entire measurement range equaled $1.718 \%$.

\section{Conclusions}

The paper presents the design and research results of the developed and practically made video-manometer. The constructed video-manometer using a flat silicone diaphragm in the full measuring range i.e. from 0 mbar to 100 mbar is a class 2. After limiting the scope to the value of 90 mbar the device has become class 1.6.

The proposed pressure sensor has the following properties: non-contact measurement; galvanic isolation between the sensor and the increased and/or decreased pressure chamber; high sensitivity, reproducibility and accuracy of carried out measurements; interference resistance; lack of frictional and mechanical components; design simplicity; small and scalable sensor sizes; the ability to measure the pressure of gases using diaphragms: flat, corrugated, bellows and capsulated; low-cost implementation; the possibility of using video fiber optics; real-time visual sensor operation self-diagnostics, virtually any number of defined alarm thresholds and methods of informing on their occurrence.

Application of the developed technique for measuring the width of the marker in conditions where the occurrence of motion blur takes place and video sensor, helped create a unique manometer (video-manometer) with a visional self-diagnostics feature executed in realtime. Self-diagnosis is carried out by analyzing the image of the measuring diaphragm obtained from the interior of the video-manometer. The developed measurement technique in sensory applications provides features unavailable for other types of transducers. It can be successfully used to build video-transmitters for other physical quantities such as distance, liquid level, temperature, etc. The presented measurement technique allows monitoring movements of the flat and corrugated diaphragm. The signal obtained directly from the sensor, without the need to convert the measured physical quantity, can be used to determine the signal error in video feedback of control systems.

\section{Acknowledgments}

The author would like to thank Prof. Tadeusz Pustelny as well as Ph.D. Eng. Grzegorz Konieczny whose publications have been an inspiration to this work.

The work was presented at the 8 th Conference Integrated Optics - Sensors, Sensing Structures and Methods, IOS'2014 sponsored by Polish Academy of Sciences. The conference was organized by the Committee of Electronics and Telecommunication at the Polish Academy of Sciences in cooperation with the Upper Silesian Division of the Polish Acoustical Society and Photonic Society of Poland, as well as the Department of Optoelectronics at the Silesian University of Technology.

\section{References}

[1] G. Konieczny, Z. Opilski, T. Pustelny, E. Maciak, Acta Phys. Pol. A 116, 344 (2009).

[2] G. Konieczny, T. Pustelny, P. Marczyński, Acta Phys. Pol. A 124, 479 (2013).

[3] G. Konieczny, T. Pustelny, Acta Phys. Pol. A 122, 962 (2012).

[4] T. Martynkien, M. Szpulak, G. Statkiewicz, G. Golojuch, J. Olszewski, W. Urbanczyk, J. Wojcik, P. Mergo, M. Makara, T. Nasilowski, F. Berghmans, H. Thienpont, Opt. Quant. Electron. 39, 481 (2007).

[5] T. Martynkien, G. Statkiewicz, M. Szpulak, J. Olszewski, G. Golojuch, W. Urbanczyk, J. Wojcik, P. Mergo, M. Makara, T. Nasilowski, F. Berghmans, H. Thienpont, Meas. Sci. Technol. 18, 3055 (2007).

[6] G. Konieczny, T. Pustelny, P. Marczyński, Acta Phys. Pol. A 124, 483 (2013).

[7] The Measurement, Instrumentation and Sensors Handbook, Ed. J.G. Webster, Taylor \& Francis Group, New York 1999

[8] H.B. Land III, C.L. Eddins, IEEE Instrum. Measur. Mag. 1094, 38 (2004).

[9] É. Pinet, Proc. SPIE 7753, 775304 (2011).

[10] I. Favero, K. Karrai, Nature Photonics 3, 201 (2009).

[11] M. Vaughan, The Fabry-Perot Interferometer: History, Theory, Practice and Applications, Taylor \& Francis Group, New York 1989.

[12] M. Szymanski, Z. Holdynski, T. Tenderenda, L. Ostrowski, M. Murawski, K. Pawlik, A. Lukowski, H. Krisch, P. Marć, L.R. Jaroszewicz, T. Nasinowski, in: Proc. 22nd Int. Conf. on Optical Fiber Sensors, Washington 2012.

[13] Z. Holdynski, M. Szymanski, T. Tenderenda, M. Murawski, L. Ostrowski, K. Pawlik, K. Gossner, H. Krisch, P. Marć, L.R. Jaroszewicz, T. Nasilowski, Proc. SPIE 8426, 84260H (2012).

[14] F. Urban, J. Kadlec, R. Vlach, R. Kuchta, Sensors 10, 11212 (2010).

[15] Y. Xiong, S.A. Shafer, in: IEEE Proc. CVPR'93, New York, 1993, p. 68.

[16] A.N. Rajagopalan, S. Chaudhuri, in: Proc. IEEE Int. Conf. on Computer Vision, 1998, p. 1047.

[17] V.P. Namboodiri, S. Chaudhuri, in: Proc. IEEE CVPR, 2008, p. 1520.

[18] K. Różanowski, K. Murawski, Acta Phys. Pol. A 122, 874 (2012).

[19] K. Murawski, R. Różycki, P. Murawski, A. Matyja, M. Rekas, Acta Phys. Pol. A 124, 517 (2013).

[20] K. Murawski, Przeglad Elektrotechniczny 9, 184 (2010) (in Polish).

[21] K. Murawski, in: Proc. XV Int. Conf. on Methods and Models in Automation and Robotics (MMAR), Międzyzdroje (Poland), 2010, p. 356.

[22] K. Murawski, K. Różanowski, Acta Phys. Pol. A 124, 509 (2013).

[23] K. Murawski, Acta Phys. Pol. A 127, 1591 (2015).

[24] K. Murawski, Acta Phys. Pol. A 128, 10 (2015).

[25] K. Różanowski, K. Murawski, Acta Phys. Pol. A 124, 558 (2013). 